difficulté n'existerait point dans un élevage spécialisé à cet effet. De plus, l'installation de cette station, étant nécessairement réalisée et conduite selon les meilleurs procédés, offrirait d'utiles exemples d'enseignement permanent.

\title{
LES SOCIÉTÉS DE PÈGHE ET LE REPEUPLEMENT DES COURS D'EAU
}

\author{
Par M le Professeur L. LÉGER \\ di lił Faculté des Sciences de l'Universilé de Grenoble, \\ Directeur de l'Institut de Pisciculture de Grenoble (1)
}

Pour les pècheurs.

pour les poissons.

Les Sociétés de Pêche dignes de ce nom ne sont plus, ne peuvent plus ètre ce qu'elles étaient simplement jadis, des groupements de bons ot joyeux drilltes qui, préférant à juste raison lie sport hygiénique et reposant che la pèche à la Jigne aux. fumées alcooliques du cabaret, avaient pour unique souci de prendre, avec agrément, le plus de poisson possible.

En effet, si l'objectif reste le même, et cela ne fait pas de doute, il laut bien reconnaître qu'aujourd'hui il est plus difficile à atteindre et, pour rispérer y arriver, le premier devoir des Sociétés est de s'employer d'abord à avoir des eaux poissonneuses. Jadis, il y avait quellques pêcheurs et beaucoup de poissons, du moins les anciens aiment à nous le dire et nous vouIons bien les croire; aujourd'hui, il y a beaucoup de pêcheurs et... quel-' ques poissous.

Les causes de cette raréfaction, qui n'est pas douteuse, sont multiples : le nombre croissant et l'habileté des pècheurs, la facilité et la rapidité des grands déplacements qui permettent d'exploiter: des régions jadis infrèquentées qui constituaient de magnifiques réserves dans les hauts bassins, les perturbations apportées au cours des caux par Jes barrages ou lies asséchements qui empèchent la montée des reproducteurs, le braconnage, les empoissonnements industriels des cours d'eau, etc. Bref, tout concourt à restreindre la multiplication, la vie même du poisson et son champ d'existence.

La rivière ne nourrit plus son homme; les vieilles familles de pêcheurs amphibies qui vivaient du produit des eaux s'éteignent sans retour, pêcheurs amateurs et professionnels se disputent et se rejettent la faute au lieu de s'entendre, ce qui serait facile avec une compréhension phus

(1) Extrait des Travaux du Laboratoipe d'Hydrobiologic et de Pisciculture de Grenołle, XXIrre amnée, igas. 
'racle des lois biologiques et économiques, et il laut vaiment que la passion de la piche soit solidement ancrée au cœur du vrai pêcheur pour voir relui-ci persister à sonder la rivière dans l'espoir incertain d'une capture incertaine.

Mais un tel état d'àme ne saurait subsister longtemps sans entrâner te découragcment et l'abandon. Aussi bien, a-t-on songé de bonne heure aux moyens à mettre en ceuvre pour relever le rendement piscicole des cours d'cau, pour prendre plus de poisson, disons le mot, malgré toutes ces eauses de raréfaction pour la plupart bien difficiles à supprimer.

C'est alors que les Sociétés de Pèche, qui avaient fléchi non pas faute de combattants, mais faute de poisson, ont pensé, à juste raison, que des efforts méthodiques cu commun, cn vne de relever le peuplement dos caux, auraient certainement plus d'action que des efforts isolés et routiniers. Et on a alors assisté à une floraison nouvelle de Sociétés de Pêchc, de groupements de Sociétés, de Fédérations, de Superfédérations, etc., qui, par leur nombre, représentent une réelle force agissante et dont les revendications justifiées méritaient d'attirer llattention des pouvoirs publics.

Le raisonnement qui a guidé et guide encore la plupart de ces sociétés nowvelles ou rajeunies est, en apparence, d'une jusle simplicité, surtout en ce qui regarde les Sogiétés de Pêche à la Truite : dès lors, pensent-elles, ¡ue l'on peut produire artificiellement des alevins de Truite, il n'y a qu'à nous en fournir et nous les déverserons dans nos rivières dépeuplées pour rendre à celles-ci leur richesse première el mème la dépasser. Ft plus nous en mettrons, pensent-elles encore, plus nous aurons de poisson.

Alor's s'engagea une véritable course à llalevin ou, plus exacteinenl, aux subventions pour achat dalevins, où les mieux placés emportaient la victoire sous forme de milliers de pauvres petits poissons plus ou moins bien portants, lancés en hâte et sans méthode dans des eaux et des endroils uqui Jeur convienment ou qui ne lcur conviennent pas, voire mème dans le grandes rivières où le courant el les ennemis de toutes sortes les déciwent en quelques jours.

Aussi bien les résultats ne furent-ils pas d'abord très brillants, au point que des voix autorisées ont pu alors linisser entendre que les repeuplemenis Gaient inefficaces. Eh bien! non, it ne faut pas dire cela. Depuis plus de Ironte ans, nous avons entrepris nous-même en Dauphiné, le repeuplement de cours d'eau à Truites appawris et disloqués par l'industrie et alors abanclonnés des pècheurs, au moyen de lancements d'alevins mesurés et méthoiliquement exécutés ; et nous pourous affirmer qu'aujourd'hui ces cours d'eau sont plius riches quils ne l'élaient avant leur période de dépeuplement. Donc le procédé est certaincment efficace; mais il ne l'est qu’à la condition d’ètre appliqué méthodiquement et rationnellement, c'est-àdire de mettre, dans les cours d'au reconnus dépeuplés, la quantiié i'alevins voulue, la qualité convenable en des endroits propices et à l'époque favorable, 
Il ne faut pas demander à la rivière plus qu elle ne peut produire of́, in conséquence, ne pas lui donner plas d'alevins qu'elle ne peut en nourvir. Si nous en mettons plus dans l'espoir d'avoir plus de poïssons, et c'est là malheurcusement le raisonnement enfantin de beaucoup de Socíslés de Pêche, nous n'aurons que des sujets de misère, décimés par la malalie, la faim ou le cannibalisme et, finallement, le résultat sera médiocre $\mathrm{d}$ souvent nul, surtout si les endroits de lancement sont mal choisis.

Donc le repeuplement a sa raison d’òtre, c'est un moven éminemmen. précieux pour relever le rendement de nos rivières; mais il ne doit être lait qu'à bon escicnt, dans les cont's d'eau où il est nécessaire, dans les points favorables et dans la mesure de la valeur nutritive, de la capacité biogénique de la rivière, el en fonction, bien entendu, de sa largeur et de lia longueur considérée.

Mais, s’il n'est pas douteux que les déverscments d’alevins méthodique. ment pratiqués sont susceptibles de ramener la richesse piscicoie dans les cours d'eau appauvris par les barrages ou autres perturbations, il ne faut pas oublicr que, dans tous les cours d'eau où les poissons trouvent encore sà et là lies conditions voulues pour se reproduire naturellement, et cest fe cas lie plus fréquent, les Sociétés de Póche ont un autre devoir à remplí pour accroître le rendement de leurs rivières.

Et celui-là, si j'ose dire, plus noble et plus en harmonie avec les lois de Ia Nature, souvent même plus cfficace et plus durable que le repeupliement artificiel ; c'est d'assurer, en le protégcant et même le favorisant, le repeu. plement naturel. Et si je reviens aujourd'hui sur ce devoir fondamental, c'est que la simplicité des déversements d'alevins, leur efficacité apparente fresque immédiate, l'a fait trop souvent perdre de vue. On n'y pense plus, or n'en parle plus. Et pourtant, pour quiconque veut assurer lie rendement nalurel de ses eaux, n'est-ce pas le premier des soins que d'entretenir, de protéger: les frayères et de favorise: l'accès aux géniteurs de ces berceau où chaque année, au débul de l'hiver, les Truites reproductrices viendront creuser le gravier pour y déposer leur précieux fardeau?

Or, tout cours d'eau, tout ruisseau qui renferme naturellement de la Truite et qui n’a pas clobstacles infranchissables vers son cours supérieur possède de tels berceaux oì s'accomplira tout seul, sans le sccours de l'Homme, Je miracle de la multiplication des poissons. Ft n'allons pas croire que cette multiplication naturelle sera forcément insuffisante pour repeupler le conrs d'eau. L'activile de deux ou trois frayères bien protŕgées suffit souvent, mảlgré le déchet naturel, à assurer et au delà le pe:iplement ammel d’une petite rivièrc. C’est par milliers que s’en échapparont des alevins autochtones, robustes, de la vraie race du pays, d'une savoureuse délicatesse, et supérieurs aux déracinés venus d'ailleurs, mème le l'étranger, et parfois tarés de maladies sursceptibles d'infester nos ruisscaux.

Lieux sacrés de la Nature, berceaux de la vie piscicole du torrent, les trayères sont des mines dor inépuisables pour lie pècheur qui sait los conscrver jalousement en les protégeant des intrus. 
Pècheurs, respect aux frayères ! Entourez-les de sollicitude et de protertion et vous trouverez bientôt votre récompense, sans autres efforts, sans nutres soucis, sans autres frais.

Pour cela, apprenez à les discerner et rotez soigneusement leur emplitcement; il est à peu près toujours le même nour un même cours d'eau ou, en tous cas, ne varie que bien peu d'une année à l'autre.

Une frayère, d'ailleurs, n'est que bien rarement isolée. Presque toujours, pllusieurs frayères se suivent ou sonl voisines, tlans une même région souvent très limitée du cours d'eau el, le plus souvent, vers son origine, sur un lond de gros gravier, dans les lètes de bassins. Ce sont les "zones à

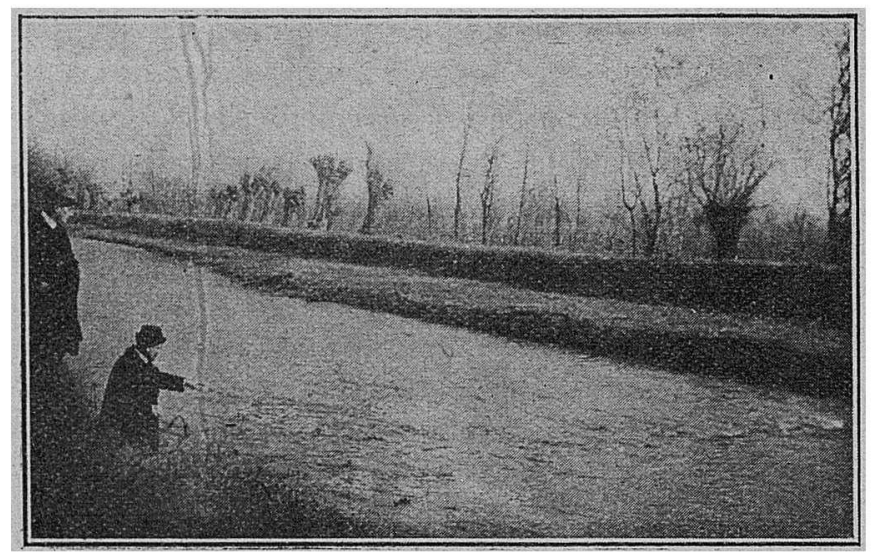

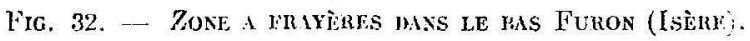

() il ilistingue, an premier plan, à leur teinte plus claire, un groune de quatre friyères de Truites.

lrayères " d'où sortira chaque année te repeuplement naturel de loul te reste du cours d'eau el mème des grandes rivières auxquelles il aboutit.

Or, chacun sait combien il est facile de capturer les reproducteurs sur l's frayères ou sur le chemin qui lles y conduit, et nul n’ignore les hécafombes annuelles qu'entraînent ces captures illicites (I). Aussi bien, le prenier clevoir d'une Société de Pèche est-il de procéder à la recherche et ¿ Ia reconnaissance de ces zones de frayères pour chacum des cours d eau ile son rayon d'action, afin de les protéger et de les surveiller au moment voulu et, au besoin, d'en faciliter l'accès aux reproducteurs.

(1) Au moment oì j’écris ces ligues surgit une lamentable preuve de ce que javance. On apporte a notre Laboratoire, aux fins d'examen, Io Truites, dont 8 femelles de plus d'un kilogramme, trouvées le ventre en l'air, dans la zone de frayères d'unc (xcellente rivière à Truites de la vallée de l'Isère. Ces sujets, qui sont, de toute évidence, les viclimes d'un empoisonnement criminel, renfermaient au lolal environ i5.0oo ocufs mûrs qui auraient cortainement donné te superbes alevins. Voici ronc un acte do liandilisme exercé sur les frayères, at fui non seulement détruit d'un coup les sujets reproducteurs, mais une progéniture parfaite et amplement sulfisante pour peupler normalement of au dela la riviere on question, sans parler do la destruction de lous lus animaux-nourriture qui donnent à ce cours d'ean unc excellente valeur piscicolo 
Une simple carte des cours d'eau du ressort de la Société indiquant la situation exacte des zones de frayères pour les principaux poissons devrait ètre dressée à cet eflet par les membres compétents fins observateurs, ct faire partic du dossier de chaque Société. On devrait même l'exiger de toute Société dè Pèche légalement constituée, désirant obtenir dẹs avantages de l'Etat.

Donc, d'abord, surveilliance et protcction des frayères dûment repérées wi moment, de la fraie dans tous les cours d'eau où les poissons, et particulièrement la Truite, peuvent ('ncore sc reproduire naturellement, et, a'autre parl, déversements rationnels d'alevins dans les cours d'eau appauvris, naturellement dépourvus de frayères ou mutilés par l'industrie. Tels sont les deux moyens à mettre en ocuvre par les Sociétés et par tous ceux qui ont lie souci d'assurer le rendement maximum de nos eaux.

La parole persuasive des Présidents ne saura jamais trop s'exercer stir les membres de leur Socićté pour en montrer l'importance, l'intérêt et la nécessité. Et les pêcheurs avisés, éclairés désormais sur le rôle respectif, l'efficacité et les résultats de ces deux mesures fondamentales : protection des frayères et repeuplement, devront s'efforcer de les appliquer à bon escient, en n'oubliant pas quc, pour les cours d'eau convenablement peu. plés qui conservent des frayères accessibles et fonctionnelles, une protection et une surveillance de quelques semaines de ces zones reproductrices suffiront le plus souvent à assurer le repeuplement maximum eî naturel de la rivière en sujets d'élite, c'est-à-dire à leur donner toute satisfactiun et agrément pour le plus grand bien du pays.

Une Société de Pêche qui protège ses frayères lorsqu'elle a le bonheur d'en posséder, qui défend ses eaux contre les empoisonneurs patentés ou non, qui déverse régulièrement à bon escient des alevins dans les cours "'eau dépeuplés privés de frayères ou barrés par l'industrie, est une Société qui remplit ses devoirs au mieux de ses intérêts et de ses aspira-. tions, mérita la confiance et la reconnaissance de tous et, en conséquence, l'appui des pouvoirs publics.

\title{
LE POISSON ROUGE
}

\author{
Par M. Marcel DAGRY
}

Le Poisson rouge ou Carassin doré est originaire d'Asie. On le trouve pius particulièrement en Chine où les habitants, passés maîtres en l'art de la déformation, en font ces monstres si élégants et si décoratifs que sont les Queues de voile et lès Télescopes.

Actuellemnt, cette variété est acclimatée dans nos pays, vit très bien dans les étangs et les pièces d'eau et s'y reproduit à merveille. Malheureusement, elle ne prend en général sa coloration qu'à l'âge de 2 et 3 ans, 